\title{
Infografía sobre actividad física para personas adultas con discapacidad
}

\section{Physical activity infographics for adults with disabilities}

\section{Palabras clave}

Discapacidad, ejercicio, salud pública, capacitismo, producción conjunta.

\section{Keywords}

Disability, exercise, public health, ableism, co-production.

\section{Javier Monforte \\ <javier.monforte@uv.es> \\ Universidad de Valencia. España \\ Joan Úbeda-Colomer \\ <joan.ubeda-colomer@uv.es> \\ Universidad de Valencia. España}

\section{Brett Smith}

Durham University. Inglaterra

\section{Charlie Foster}

<charlie.foster@bristol.ac.uk>

University of Bristol. Inglaterra

Recientemente, la revista British Journal of Sports Medicine (BJSM) ha publicado unas directrices de actividad física para personas adultas con discapacidad basadas en la evidencia (Smith, R. et al., 2018). La producción conjunta de estas directrices estuvo liderada por el Dr. Brett Smith, por encargo del Departamento de Salud Pública de Inglaterra (Public Health England). El componente central del trabajo consiste en una infografía que condensa las evidencias científicas disponibles y las presenta al público general de manera accesible y efectiva.

Crear y divulgar recursos informativos rigurosos a la par que comprensibles que promocionen estilos de vida activos en personas adultas con discapacidad es relevante y oportuno, puesto que la mayoría de estas personas no realiza suficiente actividad física para obtener beneficios saludables (Carroll et al., 2014; Úbeda-Colomer et al., 2019). En España, los recursos de este tipo son escasos, lo cual representa una barrera para que las personas

1. La presente tribuna es una adaptación traducida del artículo "Infographic. Physical activity for disabled adults" de Brett Smith et al., publicado en British Journal of Sports Medicine. Doi:10.1136/ bjsports-2018-100158.

Financiación: la investigación original fue financiada por Public Health England. Esta traducción no ha recibido financiación.

Agradecimientos: los autores agradecen a José Devís y a Víctor Pérez sus comentarios sobre una versión inicial de la traducción.

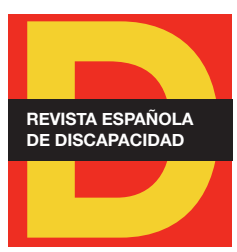

Para citar:

Monforte, J. et al. (2019): "Infografía sobre actividad física para personas adultas con discapacidad". Revista Española de Discapacidad, 7 (I): 257-265.

Doi: <https://doi.org/10.5569/23405104.07.01.14>

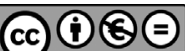


adultas con discapacidad sean físicamente activas. De hecho, la falta de información contrastada sobre aspectos de interés como la frecuencia, el tiempo y el tipo de actividades físicas necesarias para obtener beneficios saludables es una de las principales barreras que se encuentran las personas adultas con discapacidad (Rimmer et al., 2004; Williams et al., 2014).

Con el fin de acercar esta información a la comunidad hispanohablante, el presente artículo ofrece una traducción de la infografía, así como del texto y el material adicional que la acompañan. El propósito de la publicación es ampliar el alcance de la infografía para que el máximo número de personas adultas con discapacidad tenga acceso a unas recomendaciones basadas en la evidencia y pueda mejorar su salud a través de la actividad física. Así pues, invitamos a los diferentes agentes implicados en el ámbito de la salud pública a difundir la infografía mediante la plataforma que consideren más adecuada.

\section{Infografía sobre actividad física para personas adultas con discapacidad}

Pese a los beneficios de la actividad física, la mayoría de personas adultas con discapacidad en el Reino Unido no llevan estilos de vida suficientemente activos. Además, estas personas tienden a ser más inactivas en comparación con las personas sin discapacidad. Por ejemplo, un estudio reciente indicó que el $21 \%$ de personas sin discapacidad realizaban menos de 30 minutos semanales de actividad física, mientras que el porcentaje de personas con discapacidad ascendía al $43 \%$ (Sport England, 2017). Dadas las implicaciones que tiene la inactividad para la salud y la economía, promocionar la actividad física entre las personas adultas con discapacidad y reducir las desigualdades en dicha participación es una prioridad en materia de salud pública.

En 2011, los 'Principales Asesores Médicos del Reino Unido' (UK Chief Medical Officers) elaboraron las directrices para cuatro grupos de edad que comprendían desde infantes menores de cinco años hasta las personas de edad avanzada. Sin embargo, debido a la escasez de evidencias disponibles sobre la actividad física para personas con discapacidad, las directrices omitieron pautas para este colectivo. En 2018, el Departamento de Salud Pública del Reino Unido nos encargó revisar las evidencias y, en el caso de que estas resultasen ser suficientes, producir conjuntamente unas recomendaciones sobre actividad física y discapacidad en un formato apropiado.

En la revisión se encontraron múltiples beneficios de la actividad física para personas adultas con discapacidad, como por ejemplo la mejora en la calidad de vida (Smith, R. et al., 2018). A partir de las evidencias, se recomienda que las personas adultas con discapacidad realicen actividades de fuerza y equilibrio, al menos dos días por semana. Aunque un poco de actividad física es mejor que nada, se recomienda que las personas adultas con discapacidad aspiren a realizar al menos 150 minutos de actividad física de intensidad moderada por semana para obtener mejoras sustanciales en la salud (Smith, R. et al., 2018). No se encontraron evidencias de que la participación en actividades físicas apropiadas incrementara el riesgo de lesión o daño (Smith, R. et al., 2018).

A partir de la revisión, trabajamos con cerca de 350 personas adultas con discapacidad, 10 organizaciones dedicadas a la discapacidad y 50 profesionales de la salud con el fin de explorar cómo se podría producir 
conjuntamente un recurso accesible y adecuado que permitiera presentar las recomendaciones de actividad física basadas en la evidencia al público de interés. La producción conjunta se sitúa en el centro de iniciativas recientes de investigación, conocidas como 'Implicación Pública del Paciente', 'Medicina Participativa' y 'Ciencia Ciudadana'. Durante varios talleres presenciales, las personas adultas con discapacidad, organizaciones y profesionales de la salud plantearon que la gratificación (por ejemplo: la actividad física te hace sentir bien) y el sedentarismo deberían ser incluidas en los mensajes sobre actividad física, mientras que debería evitarse un énfasis distorsionado hacia la capacidad física (capacitismo) (Smith, R. et al., 2018). El capacitismo denota un prejuicio, en muchas ocasiones irreflexivo, que beneficia a las personas sin discapacidad y discrimina a las personas con discapacidad (Goodley, 2014). Así se observa en mensajes como "Levántate, siéntate menos" o "Siéntate menos, muévete más", que favorecen ciertos cuerpos (por ejemplo: aquellos que pueden levantarse o evitar sentarse fácilmente) al tiempo que discriminan otros (por ejemplo: usuarios de sillas de ruedas).

Las personas adultas con discapacidad, organizaciones y profesionales de la salud también identificaron las mejores formas para comunicar las recomendaciones. Estas incluían:

- Campañas mediáticas como 'This Girl Can',

- Recursos web, y también

- Infografías dirigidas al público con discapacidad.

Se consideró que una campaña intersectorial en los medios de comunicación, que evitara representar a las personas con discapacidad únicamente como 'súper héroes', podía resultar un recurso inspirador para que las personas con discapacidad se convirtieran en personas activas.

Los recursos web se consideraron útiles para identificar qué tipos de actividad física hay disponibles en la comunidad local y dónde pueden realizarse esas actividades en un ambiente inclusivo. No obstante, existía la preocupación de que la información de internet pudiera estar desorganizada, resultar poco fiable, estar obsoleta o ser inaccesible. Otra limitación que se apuntaba fueron los costes financieros de producir y mantener una web con recursos y campañas mediáticas.

En cambio, las infografías se consideraron recursos de coste relativamente bajo y también singularmente adecuados para difundir y transmitir recomendaciones sobre actividad física. Este enfoque se ha utilizado en las directrices de los 'Principales Asesores Médicos del Reino Unido' en 2011 (Smith, R. et al., 2018). Las personas adultas con discapacidad, organizaciones y profesionales de la salud juzgaron que una infografía podría comunicar información compleja de una forma asequible, comprensible y sugerente a un número elevado de personas. Prácticamente todos pensaban que las infografías podían concienciar, cuestionar actitudes e inspirar a las personas con discapacidad para cambiar su conducta y empezar a ser más activos físicamente (Smith, B. et al., 2018). Los participantes añadieron que estos beneficios podrían ser mayores si se incluía a mensajeros clave para promocionar la actividad física. Entre los mensajeros identificados, por orden de importancia, estaban las personas adultas con discapacidad, profesionales del trabajo social, profesionales de la salud, organizaciones de actividad física, deporte o dedicadas a la discapacidad, y personas encargadas de las actividades de ocio.

Se propuso que estos mensajeros de confianza combinaran distintos tipos de infografías para promocionar la actividad física de forma extensa y continua. Lo cual podría hacerse por medio de folletos impresos para 
entregar en mano, por correo electrónico, mediante redes sociales o páginas web y también en forma de póster para colgar en espacios públicos como consultas de medicina general, instalaciones de servicios sociales y centros de ocio. Así pues, se acordó que la infografía debía ser el producto final que derivara de la producción conjunta (figura 1). El proceso de elaboración de la infografía se detalla en el suplemento que acompaña al presente texto.

Esta es la primera infografía producida conjuntamente con el propósito de comunicar recomendaciones de actividad física basadas en la evidencia a un conjunto heterogéneo de personas con discapacidad. Ha sido aprobada por el Departamento de Salud Pública de Inglaterra y respaldada por los 'Principales Asesores Médicos del Reino Unido'. La infografía nos parece un recurso adecuado para la acción coordinada y sirve de anticipo a la actualización de las directrices vigentes de actividad física de los 'Principales Asesores Médicos del Reino Unido' que saldrán en 2019. Esperamos que se comparta, se visibilice y se utilice para promocionar la actividad física y combatir las desigualdades. 
Figura 1. Infografía sobre actividad física para personas adultas con discapacidad

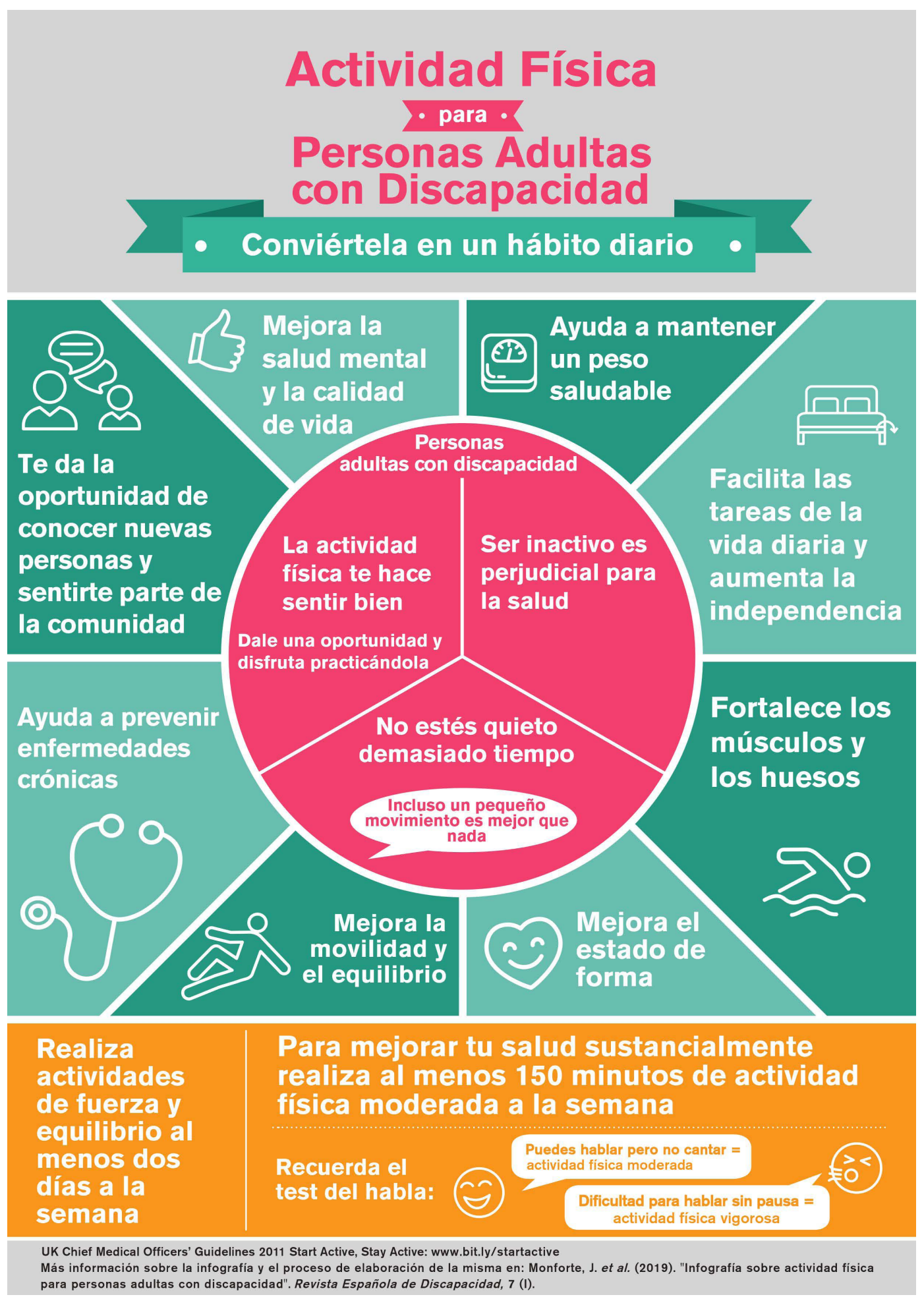




\section{Referencias bibliográficas}

Carroll, D. D. et al. (2014): "Vital signs: Disability and physical activity-United States, 2009-2012". MMWR. Morbidity and mortality weekly report, 63 (18): 407-413.

Goodley, D. (2014): Dis/ability studies: Theorising disablism and ableism. Londres: Routledge.

Rimmer, J. H. et al. (2004): "Physical activity participation among persons with disabilities: Barriers and facilitators". American journal of preventive medicine, 26 (5): 419-425.

Smith, B. et al. (2019): "Infographic. Physical activity for disabled adults". British Journal of Sports Medicine, 53: 335-336 (en línea). <https://doi.org/10.1136/bjsports-2018-100158>, acceso 20 de noviembre de 2018.

Smith, B. et al. (2018): "Physical activity for general health benefits in disabled adults: Summary of a rapid evidence review for the UK Chief Medical Officers' update of the physical activity guidelines". London: Public Health England.

Smith, R. et al. (2018): "CMO physical activity expert committee for physical activity and pregnancy". British Journal of Sports Medicine, 52: 532-533 (en línea). <https://doi.org/10.1136/bjsports-2017-098037>, acceso 20 de noviembre de 2018.

Sport England (2017): Active Lives Adult Survey (en línea). <https://www.sportengland.org/media/12458/activelives-adult-may-16-17-report.pdf>, acceso 20 de noviembre de 2018.

Úbeda-Colomer, J. et al. (2019): "Physical activity of university students with disabilities: accomplishment of recommendations and differences by age, sex, disability and weight status". Public Health, 166: 69-78 (en línea). <https://doi.org/10.1016/j.puhe.2018.10.006>, acceso 20 de noviembre de 2018.

Williams, T. L. et al. (2014): "The barriers, benefits and facilitators of leisure time physical activity among people with spinal cord injury: A meta-synthesis of qualitative findings". Health Psycholgy Review, 8 (4): 404-425. 
Anexo 1. Material adicional: proceso de elaboración de la infografía

La discapacidad hace referencia a las personas con una deficiencia física (por ejemplo lesión medular), sensorial (por ejemplo deficiencia visual), cognitiva (por ejemplo dificultades de aprendizaje) y/o mental (por ejemplo depresión) que, en interacción con diversas barreras, puede dificultar la participación social plena, efectiva y en igualdad de condiciones con los demás. En el proceso de producción conjunta de la infografía participaron personas adultas con deficiencias físicas, sensoriales, cognitivas y/o mentales. La producción conjunta de la infografía, así como algunas ideas obtenidas a lo largo del proceso, pueden resumirse de la siguiente manera:

1. Las evidencias generadas a partir de la revisión son fruto de la colaboración con personas adultas con discapacidad con diferentes grados de participación en actividades físicas (de muy inactivas a muy activas), profesionales sanitarios y organizaciones dedicadas a la discapacidad. El trabajo realizado consistió en resumir los principales beneficios de la actividad física, la frecuencia, la intensidad, el tiempo y el tipo de actividades que las personas adultas con discapacidad necesitan para obtener beneficios para su salud, así como cualquier riesgo vinculado con la actividad física y las consecuencias negativas de la conducta sedentaria.

2. En colaboración con las personas adultas con discapacidad y las organizaciones, se revisaron las estrategias comunicativas que en la actualidad resultan relevantes para las personas adultas, tanto en el Reino Unido como en el extranjero.

3. La realización de tres diseños preliminares se enmarcó en unas instrucciones básicas establecidas de manera colaborativa. Los tres diseños se basaban en la misma evidencia. Entre los desafíos que surgieron durante este proceso se encontraban evitar mensajes densos con mucho texto, mantener la claridad del mensaje y encontrar un diseño gráfico que concentrase los mensajes clave y a la vez ayudara a 'guiar' a los/as lectores/as a través de la información. Otro de los retos recaía en la forma de comunicar la intensidad de la actividad física de forma efectiva. A este respecto, se sugirieron el icono visual de una batería, un termómetro y el test del habla. Por otra parte, se llegó a un consenso unánime: la infografía no debía centrarse en un solo tipo de discapacidad (por ejemplo personas con lesión medular) sino, por el contrario, promover la inclusión, la conectividad y la diferencia de manera que todos los tipos de discapacidad estuviesen representados. En esta línea, se concluyó que el alcance de la infografía sería mucho más amplio si se dirigía a las personas adultas con discapacidad en lugar de a un solo tipo de discapacidad.

4. Las personas adultas con discapacidad, los/as profesionales sanitarios, las organizaciones y el equipo de expertos/as discutieron, analizaron y revisaron los diseños iniciales en talleres presenciales o en la red. Esto resultó en la modificación y realización de un único diseño. Si bien el test del habla se consideró la mejor opción para comunicar la intensidad, llegar a un acuerdo sobre qué otros iconos visuales incluir y excluir resultó un desafío. En este sentido, las personas adultas con discapacidad sugirieron que debían evitarse los iconos visuales que representaran la discapacidad, como por ejemplo el de una persona en una silla de ruedas. Por otra parte, algunas personas adultas con discapacidad opinaban que debían usarse dos mensajes para comunicar la frecuencia de la actividad física. En particular, se plantearon 'para mejorar tu salud sustancialmente realiza al menos 150 minutos de actividad física moderada 
cada semana' y 'para conseguir algunos beneficios saludables realiza al menos 60 minutos de actividad física moderada cada semana'. La posibilidad de incluir dos mensajes en lugar de uno se tuvo en cuenta porque algunas personas con discapacidad físicamente inactivas pensaban que 60 minutos constituye una meta más realista y motivante que 150 minutos. No obstante, la meta de los 150 minutos se consideraba esencial para comunicar las ganancias sustanciales de salud que acompañan a una mayor práctica de actividad física. También se consideraba una meta motivante para aquellos que prefieren realizar estiramientos en solitario o acumular actividad física a lo largo de la semana. A pesar del potencial que presenta la incorporación de dos mensajes, lo que representa un giro hacia la relación dosis-respuesta, también se tuvo en cuenta que añadir un mensaje con menos frecuencia (por ejemplo 60 minutos) crearía confusión en el público por lo que respecta a la cantidad de actividad que debería realizarse para obtener beneficios reales en el estado de salud. Cabía la posibilidad de que la confusión comprometiera el éxito en la promoción de la actividad física. Por tanto, solo se mantuvo el mensaje de 150 minutos. Con todo, esta cuestión debe permanecer sujeta a revisión. Si la evidencia disponible fuera suficiente, en mensajes futuros habría que incluir un énfasis en la relación dosis-respuesta para la salud. Otra de las ideas clave que se obtuvieron a partir del proceso de producción conjunta fue la gran relevancia de la gratificación (por ejemplo diversión, disfrute, placer) en la comunicación. Por tal motivo, los mensajes relacionados con el afecto se reubicaron, pasando del final de la infografía a una posición más central dentro de la misma. Como ya se ha indicado anteriormente, se subrayó la importancia de la conducta sedentaria y el capacitismo. Las personas adultas con discapacidad y las organizaciones se mostraron de acuerdo en que los mensajes acerca del sedentarismo eran esenciales, aunque consideraron que ciertos mensajes, como "Levántate más" o "Siéntate menos", deberían evitarse. Estos mensajes son discriminadores y, lejos de ser inclusivos, reflejan un prejuicio irreflexivo al contemplar solamente a los cuerpos 'capaces' cuando se habla de conducta sedentaria. Por tanto, estos mensajes se evitaron. También se recomendó que cualquier futuro mensaje sobre el sedentarismo tuviera un carácter inclusivo.

5. La infografía elegida se discutió, se analizó y se revisó en otra tanda de talleres presenciales, o bien vía online, con las mismas personas adultas con discapacidad, profesionales sanitarios, organizaciones, el equipo de expertos/as y dos miembros del Departamento de Salud Pública de Inglaterra. A partir del feedback obtenido en estas reuniones, hubo más modificaciones en el diseño. Los cambios incluyeron una modificación sobre el mensaje original relativo a la seguridad en la práctica de actividad física, que se cambió a "Ser inactivo es perjudicial para la salud". Asimismo, se modificaron dos nuevos iconos, puesto que no resultaban comprensibles para algunas personas con trastornos mentales. Como ya había ocurrido en ocasiones anteriores, algunas personas sugirieron cambios o ampliaciones a la infografía que no se llevaron a cabo debido a la falta de evidencias que las apoyaran. A menudo, algunas personas se sentían decepcionadas cuando se les explicaba la razón por la que no se podía realizar un cambio determinado o añadir un mensaje. Con todo, se consideró necesario fundamentar la infografía en la evidencia.

6. Las personas adultas con discapacidad, las organizaciones, los/as profesionales sanitarios y el equipo de expertos/as dieron feedback adicional sobre la versión actualizada de la infografía en siguientes talleres presenciales, vía online o por teléfono. A raíz de sus comentarios, la infografía se modificó ligeramente.

7. La infografía se presentó al Departamento de Salud Pública de Inglaterra para contar con su valoración. Se sugirieron cambios menores. Por ejemplo, se propusieron pequeñas modificaciones en el test del habla. 
8. Las personas adultas con discapacidad, los/as profesionales sanitarios y el equipo de expertos/as revisaron el feedback del Departamento de Salud Pública de Inglaterra, y se realizaron pequeñas correcciones atendiendo al mismo. Se enfatizó en la importancia de traducir la infografía al sistema braille.

9. El Departamento de salud Pública de Inglaterra, las personas adultas con discapacidad, los/as profesionales sanitarios, las organizaciones dedicadas a la discapacidad y el equipo de expertos/as revisaron la infografía editada y la aprobación del Departamento de Salud Pública de Inglaterra puso el punto final al proceso de elaboración. También se envió la infografía a los 'Principales Asesores Médicos del Reino Unido' para su aprobación.

10. La infografía fue aprobada y respaldada por el Departamento de Salud Pública de Inglaterra y los cuatro 'Principales Asesores Médicos del Reino Unido'. Se decidió publicar la infografía en una revista científica con el fin de difundirla a la audiencia académica. Para llegar al público general, el Departamento de Salud Pública de Inglaterra lanzó una infografía el 15 de octubre de 2018, con una cobertura mediática. La infografía aparece en la página web 'Start Active, Stay Active'2.

Otras actividades de difusión en colaboración con organizaciones dedicadas a la discapacidad, profesionales sanitarios, trabajadores/as sociales y asociaciones de atención social, organismos deportivos, programas comunitarios y servicios educativos, entre otros, se encuentran en vías de desarrollo.

2. Más información: https://www.gov.uk/government/publications/start-active-stay-active-a-report-on-physical-activity-from-the-four-homecountries-chief-medical-officers. 